\title{
Investigation of DLP Projector as an Energy Light Source to Cure Silver Conductive Ink
}

\author{
Rd Khairilhijra Khirotdin ${ }^{1, a}{ }^{*}$, Adham Mohamad Nasir ${ }^{1, b}$, Khairu Kamarudin ${ }^{1, c}$, \\ Mustaffa Ibrahim ${ }^{1, d}$ \\ ${ }^{1}$ Faculty of Mechanical Engineering and Manufacturing \\ Universiti Tun Hussein Onn Malaysia
}

akhairil@uthm.edu.my, bajangpro@gmail.com, ${ }^{\text {}}$ khairu@uthm.edu.my, ${ }^{\text {}}$ mustaffa@uthm.edu.my

Keywords: Alternative Energy Light Source, Curing Process, DLP Projector, Silver Conductive Ink.

\begin{abstract}
Conductive ink has been widely used in many application of electronic parts. It normally comes in liquid form and will need to be cured usually by conventional oven or laser irradiation after drawn to expose its metallic contents. But, these curing processes suffer from numerous problems which is costly process and warping defect when cured using conventional oven. Thus, alternative process is prominent and DLP projector has been seen capable of polymerised photopolymer materials. By making use of its potential associated with a proper control of the light intensity, exposure time and distance, it could provides sufficent heat to cure conductive ink. This study investigates the curing process of silver conductive ink on polymer substrate using DLP projector light with a variation of curing time and distance. Electrical resistance, hardness and adhesion level of cured sample were measured to determine its functionality. The conductive ink is deposited on the substrate using doctor blade method and Microsoft Power Point slide is used to shape the light image produced by the projector. It was found that the conductivity and curing level of the sample cured were higher at the distance which getting the sharpest light image. The performances of the samples were also greater as the curing time increased. DLP projector is able to cure the conductive track efficiently with shorter curing time apart from the cheap preparation and setup cost.
\end{abstract}

\section{Introduction}

Conductive ink has been widely used in many applications of electronic parts [1]. One of the famous applications is RFID (Radio Frequency Identification) tag. RFID uses radio frequency electromagnetic fields to transfer data without any contact to identify and track the tags attached to the regarding items. RFID tags are used in a lot of industries [2]. RFID tag contains an integrated circuit which is usually made from conductive coating or ink tracks. The coating and ink originally came in liquid form and will be drawn on a substrate and cured afterwards. The ink drawn on the substrates are usually cured with oven, microwaves and lasers for non-thermal curing method [3]. These processes use heat to evaporate binders and unwanted solvent in conductive ink polymers leaving only the metallic content on the substrate that is already been hardened and sticks onto the substrate. The conventional curing methods have their own weaknesses which laser irradiation process is a costly process [4] and silver conductive ink cured with conventional oven might undergo a warping defect [5]. Thus, there is a need to explore the possibility of using DLP projector as it was proven before that DLP projector had succesfully polymerised a photopolymer materials [6]. Making use of its potential associated with a proper control of the light intensity and exposure time and distance, it could provides sufficent heat to cure conductive ink. Furthermore, by using DLP projector the cost of curing process will be decreased since it is cheap, easy to be set up and portable. With the wide use of the technologies, the production time and cost become two apparent elements which reflect the effectiveness of the technologies. Hence, this study is to investigate the 
possibility of using DLP projector as another source of heat to be used in curing silver conductive ink track.

Limitations of conventional curing methods. Curing is a process to crosslink the polymer materials. A good composition can be formed that exhibit physical properties such as high tensile strengths, low compression set, recoverable elongations, high tear energies, and improved dynamic performance. One of the curing methods is using oven. The oven produced heat to melt the material which evaporates the binders and unwanted solvent in the material to harden and as well cross linked with the substrate. However, there were limitations in which when the flexible and cheap substrate is used, they might physically deform due to the heat exposed. The conductivity at lower cure temperatures is adequate for some applications, but it is still in the range of $120^{\circ} \mathrm{C}$ to $250^{\circ} \mathrm{C}$ meaning cheap polyester film and paper cannot be used without warping in the cure ovens. Laser curing is better than oven, as the thermally cured sample had lower electrical resistivity [3]. However, the case was attributed to the time difference between curing methods which took two seconds for the laser curing and an hour for thermal curing. But on silver based conductive ink, laser has difficulty penetrating the ink to the base because of the reflectivity of the material itself [7]. Microwave is also been used as a curing method via Variable Frequency Microwave (VFM) where it uses a variable swept frequency to apply microwave energy into a closed chamber for curing. Polymer resins, containing polarized molecules, will couple and absorb with the microwave energy, causing rapid polymerization of the resins as the energy is dissipated in the form of heat. Since the frequency is rapidly swept, arcing problems associated with metal or semiconductor components is eliminated. ZArcing is the result of excessive charge build-up in metallic materials in the presence of standing wave patterns. In the VFM technique, the electric fields are electronically stirred and the microwave energy is not focused at any given location more than a fraction of a second. The dynamics of charge build-up that leads to arcing are never achieved. Hence, bare semiconductor devices are not adversely affected by the microwave energy, resulting in defect-free processing. Hot plate curing method also been employed where it uses a plate to transfer heat to the substrate and polymer. The substrate is heated to certain temperature on the hot plate during the deposition process to improve deposit quality. A hot plate produces heat by using electricity through its heat coils, instead of traditional means of producing heat by using fire. The heat coils have a relatively high level of electrical resistance. As electricity encounters resistance along its course, the buildup changes from electrical energy to heat energy cause the heating coils to emit heat. In a paper which used ink-jet printing, the liquid in the droplets was flash-evaporated on contact, halving the feature size in comparison to room temperature printing by heating the substrate on the hot plate [8].

Conductive ink and coating. Particulate silver inks are increasing in the use for direct manufacture of electronic circuitry than the traditional method [9]. Silver based conductive ink is the most effective conductive ink. Silver has great conductivity and anti-oxidation properties, thus silver based inks have attracted a lot of attention [4, 5, 7]. It is made of mostly silver (about $45-55 \%$ of the total solution). This percentage insures the right viscosity so that the ink flows without leaking or clogging. Also, it can be written on soft and hard surfaces. The other $45-55 \%$ is the fluid solution composed of Diethanolamine and Hydroxyethyl cellulose. Silver based conductive ink has high conductivity since it is made mostly of silver flakes and particles. The resistivity will even become lower after the drying process. It is also flexible that the paper on which silver based conductive ink circuit has been printed on can be folded and bent many times without affecting the conductivity. Due to the expensive price of silver, silver based conductive ink is not very popular with the general public. The common application that used the silver based conductive ink is the RFID tag, antennas and few others.

Substrate. Substrate is the surface that the conductive ink is deposited onto. There are a few common substrates used in the DW technology nowadays. The popular types include alumina, polyethylene terephthalate (PET), carbon fiber composite (CFC), polyaramid and paper. Alumina is used as a well known dielectric material, which is chemically inert and thermally resistant; it also has excellent durability in harsh environments due to its hardness. Alumina is light weight and the 
price is reasonably cheap. PET is preferable for its light weight, physical flexibility, elasticity against stress, and economical advantage. However, it was found that the PET material is hard to be obtained in small quantity. Therefore, Cellulose Acetate material has been chosen considering its properties and flexibility. It is famously known as OHP film and it has $0.167 \mathrm{~W} / \mathrm{mK}$ of thermal conductivity, $1280 \mathrm{~kg} / \mathrm{m} 3$ in density, and $1451 \mathrm{~J} / \mathrm{kgK}$ in specific heat capacity. It is cheap, affordable, and the properties of the OHP film are almost similar to the PET properties.

\section{Methodology}

The experiment generally starts with the deposition of the ink track onto a polymer substrate using a doctor blade technique [4]. The ink track samples were then transferred onto the modified stereo lithography apparatus for curing before hardness and resistivity measurements are carried out.

Deposition method. A doctor blade method is used to deposit the ink in which a metal stencil and a low tack tape has been applied on polymer substrate. The stencil is placed on the taped polymer substrate and a knife is used to cut the tape accordingly leaving a negative shape of the track image. The stencil is then removed and the negative track of the tape was then flooded with ink and a scraper is used to draw across the surface of the tape. Once it had been properly flooded and scraped with excess ink removed, the tape was peeled off leaving a positive image of ink as shown in Figure 1. The geometry of the track is a single line with a dimension of $70 \mathrm{~mm}$ length with a $2.8 \mathrm{~mm}$ width. Figure 2 shows the stencil dimension in millimeters.

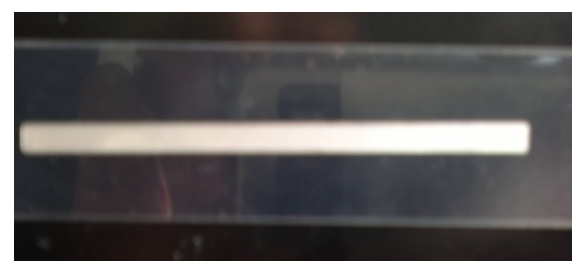

Fig. 1 Positive image of ink track on substrate

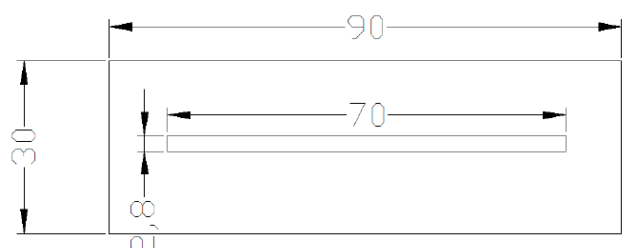

Fig. 2 Stencil dimension

Preparation of PowerPoint slide. Microsoft PowerPoint has been used as a CAD system to produce the light image from the projector. A new blank slide is opened and the background of the slide is then set to black. A simple long squared shape is drawn with white color. The length of the shape represents the length of the exposed light on the platform. The width of the shape represents the width of exposed light on the platform. The adjustment to the width of exposed light on the platform has been done to suit with the track length and width.

Curing process. The sample is placed on the platform of stereo lithography apparatus. The distance between platform and the optical lens and the distance between the optical lens and DLP projector are explained in detail in the experiment parameters section. The sample is placed accordingly to ensure the light image could be pointed properly on the ink track. The projector is pre-heated prior to the curing process to ensure the heat that came out from the projector's bulb is in stable hot condition.

Manual Scratch Test. After the samples were cured, it will be manually scratch tested to examine its cure level. The test is performed using a needle. A uniform load has been applied while scratching the needle onto the ink track. If the ink track had any smear, the sample is considered as not fully cured and the next procedure will not be performed.

Hardness Test. The test has been performed using the micro hardness tester. A load will be subjected on the indenter to penetrate the cured ink track. The indentation produced a square based mark on the ink track surface. The mark is called diagonal and the diagonal length of the indentation indicates the cured level and they are obtained from the average of D1 and D2 as in Figure 3.

Adhesion Test. To perform the test, one part of the ink track will be cut into 100 small squares with knife. The $3 \mathrm{M}$ tape is glued onto the squares area (crosshatch) as shown in Figure 4 . The tape is smoothed into place using a pencil eraser over the area of the incisions. The tape is then removed 
by pulling it off rapidly close to $180^{\circ}$. The adhesion level of the ink track is assessed based on its classification table from $0 \mathrm{~B}$ to $5 \mathrm{~B}$.

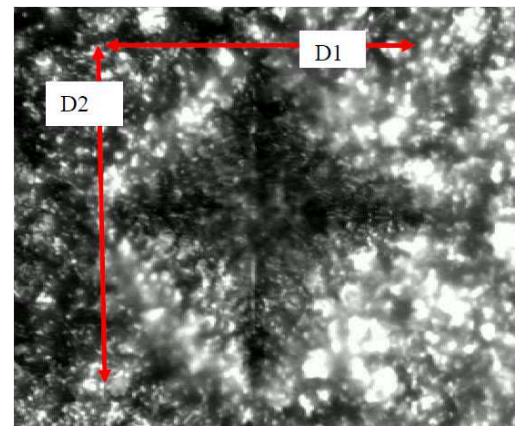

Fig. 3 Indentation on silver conductive Ink track

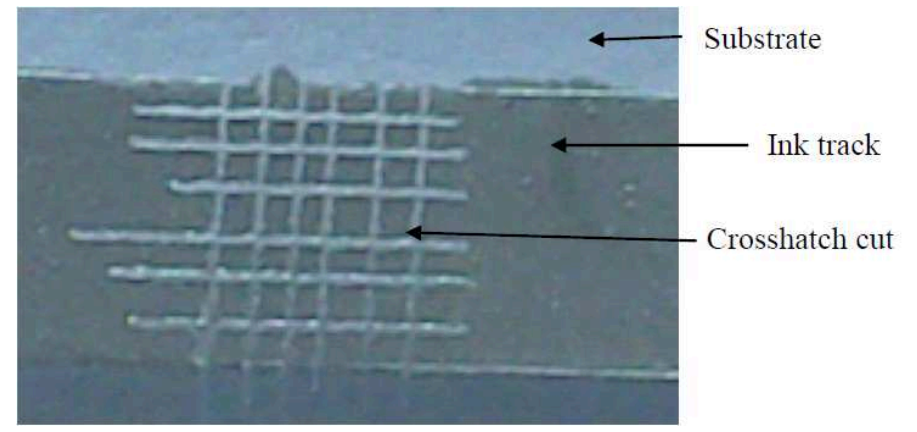

Fig. 4 Crosshatch cut on ink track

Resistance measurement. In order to measure the resistance of the cured ink, the probes are held in firm contact with the ink track as shown in Figure 5, and the resistance value is shown on the display panel.

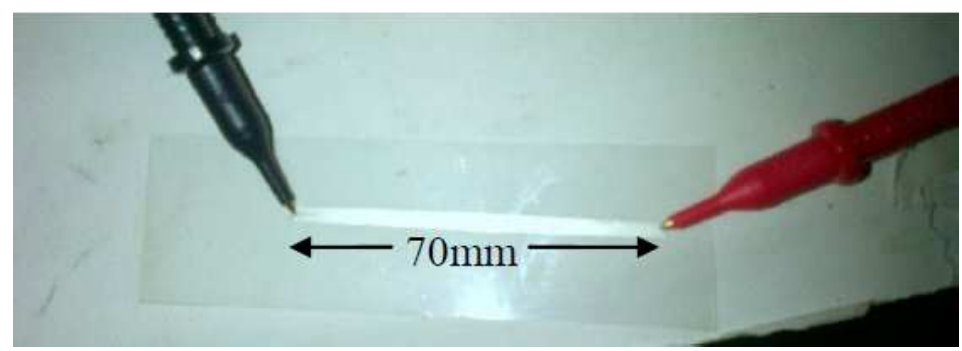

Fig. 5 Resistance measurement

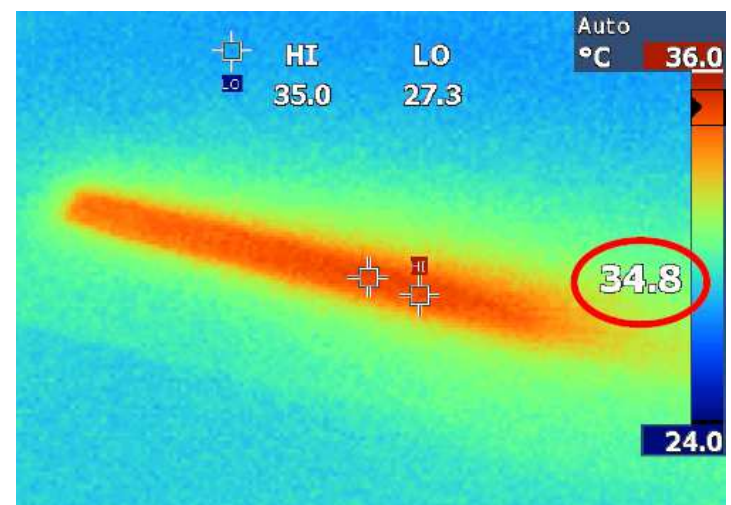

Fig. 6 Thermal image of the ink track while curing

\section{Results}

Temperature measurement. A thermal imager is used to capture a thermal image of the ink track during curing process. A temperature reading is taken to observe the thermal effect of the light projected to the ink track. Fig. 6 shows the image of the ink track captured by the thermal imager while undergoing curing process. The temperature readings on the ink tracks rose as the curing time increased. The ink track which is cured at a distance of $165 \mathrm{~mm}$ shows the highest inclination compared to the ink track cured at $220 \mathrm{~mm}$ and $110 \mathrm{~mm}$ distance. The ink track cured at $165 \mathrm{~mm}$ distance also had a maximum temperature at almost all of the curing time. This shows that curing distance is not the appropriate reason for the inclination of the temperature readings.

Resistance measurement. Fig. 7 shows the result of the resistance along the ink track cured at $165 \mathrm{~mm}$ which it has the lowest maximum and minimum values as to compare with the ink track that is cured at $110 \mathrm{~mm}$ and $220 \mathrm{~mm}$ distance between the lens and platform. 


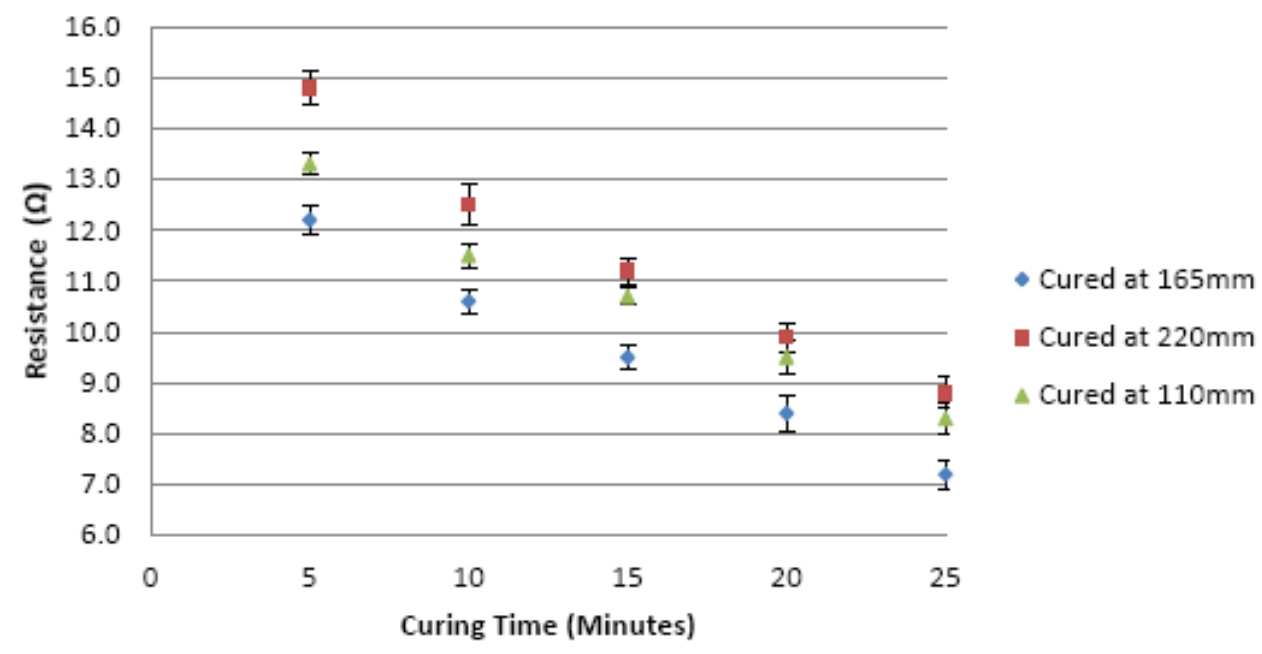

Fig. 7 Average resistance against curing time at a variable curing distance

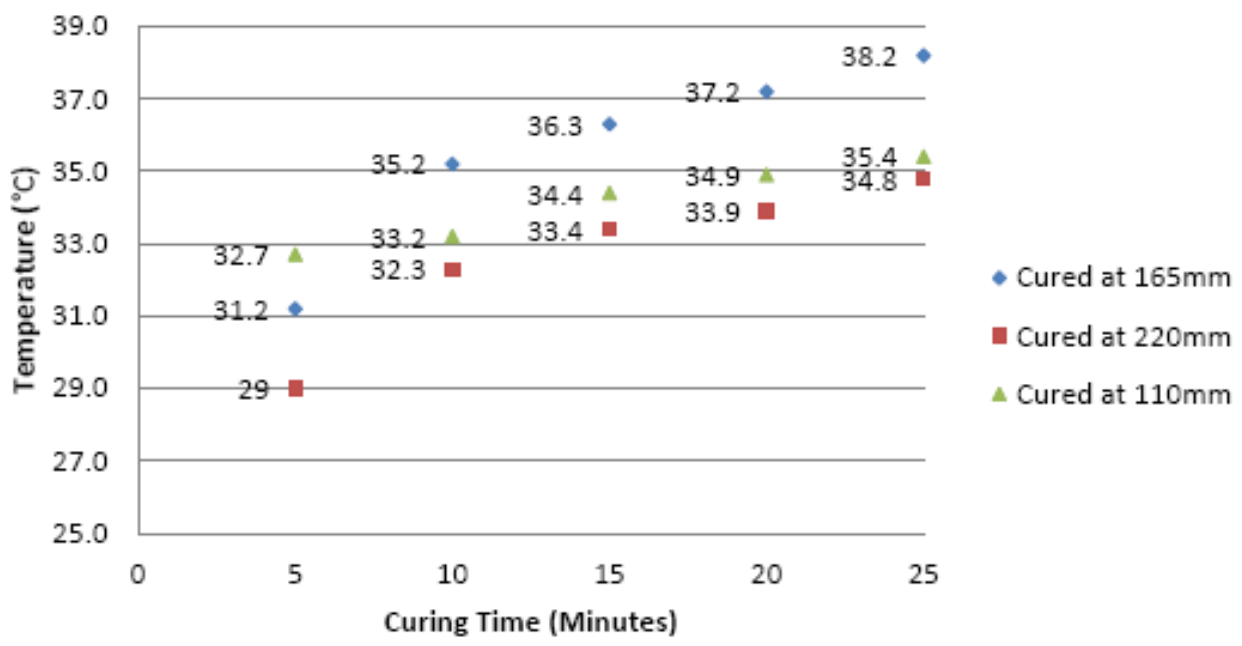

Fig. 8 Temperature against curing time at a variable curing distance

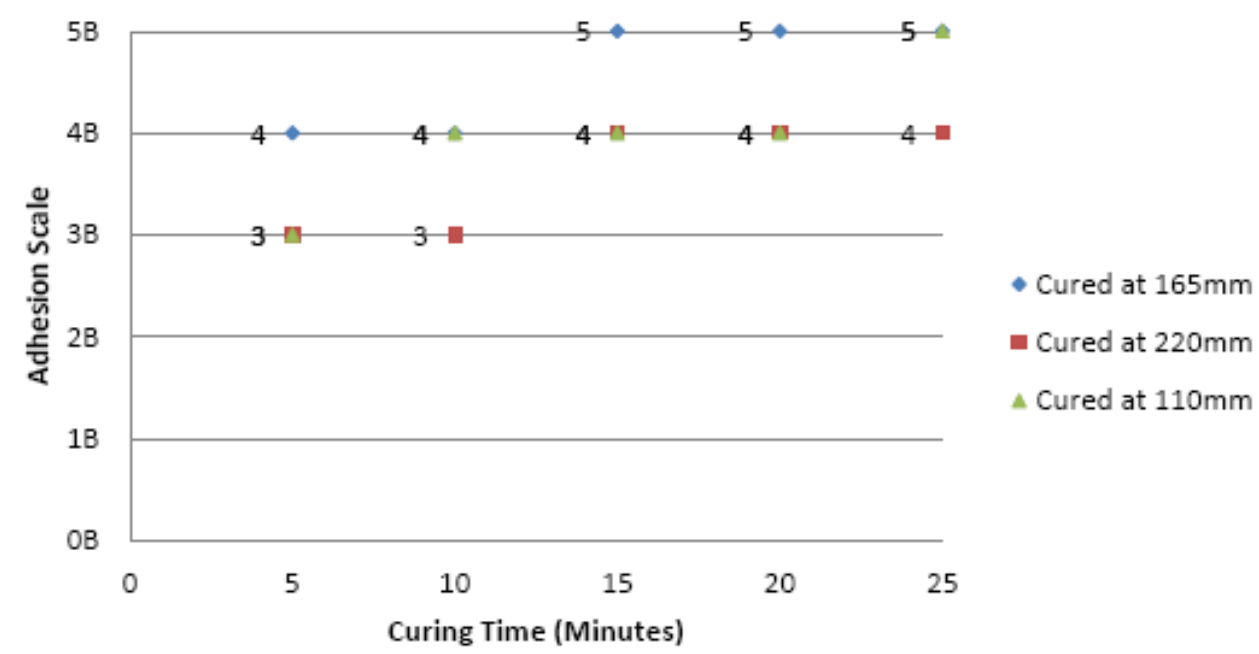

Fig. 9 Adhesion scale against curing time at a variable curing distance

Tape test. A tape test is conducted on the sample to measure its adhesion level. The crosshatch pattern on the ink track is applied with the tape test. The crosshatch is compared based on the percentage of ink particulate removed. The adhesion level is assessed based on 0B to 5B scale. Fig. 9 shows the graph of adhesion scale of each curing distance as the curing time increased. The 
adhesion of the ink track shows an inclination trend as the curing time increased. The ink track cured at $165 \mathrm{~mm}$ distance shows the highest adhesion compared to the ink track cured at $110 \mathrm{~mm}$ and $220 \mathrm{~mm}$.

Hardness test. A hardness test is conducted to determine the ink cured hardness level at a variation of time and distance. It was found that the suitable test load is HV0.05 which is equivalent to $490.3 \mathrm{mN}$. The smaller load made almost no indentation to the ink track and the higher load is not necessary. Fig. 10 shows that the hardness of the ink tracks increased as the curing time increased. The ink track cured at $165 \mathrm{~mm}$ distance had a higher hardness value compared to the ink tracks cured at $110 \mathrm{~mm}$ and $220 \mathrm{~mm}$ distance.

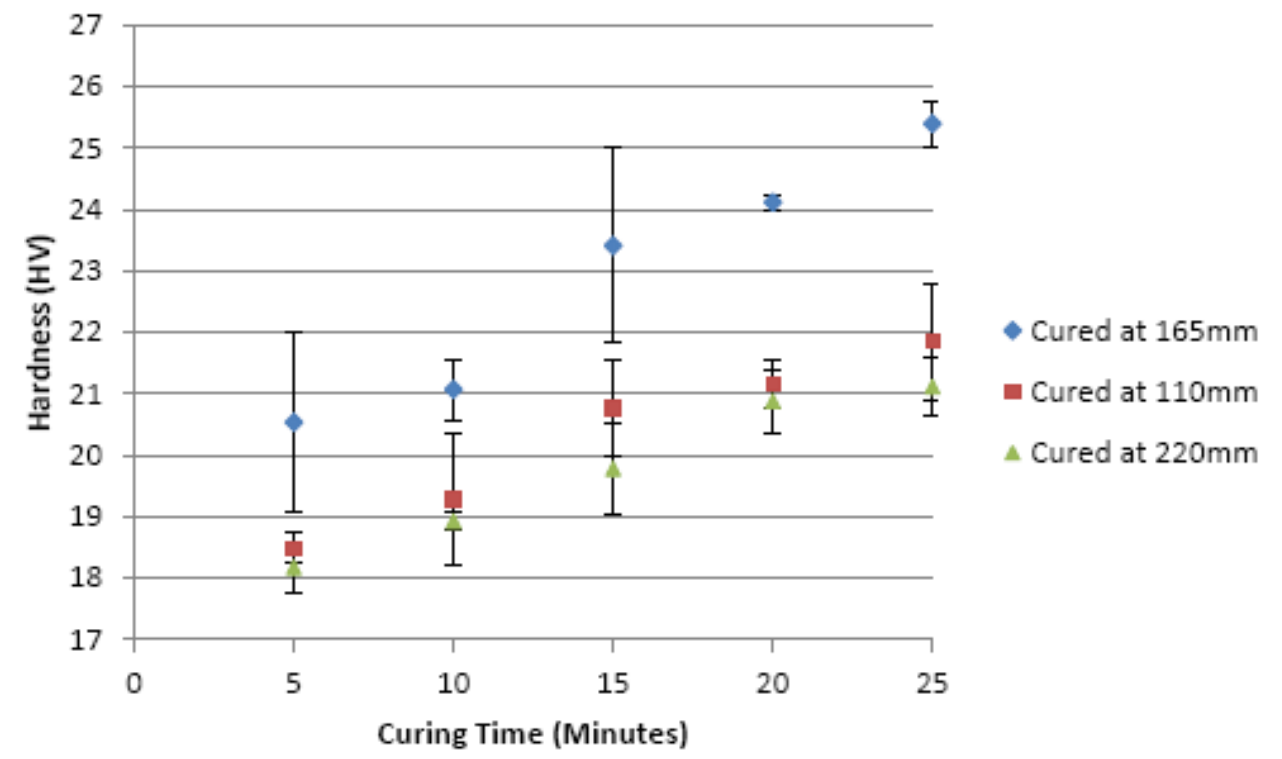

Fig. 10 Hardness against curing time at a variable curing distance

\section{Discussion}

Variation of curing distance. At a distance of $165 \mathrm{~mm}$ between the lens and platform, the light image was at its sharpest edge. It can be said that the light of the projector is having its highest intensity and brightness to provide the silver conductive ink track with sufficient energy and heat. The light image on the other two distances between the lens and the platform which is at $110 \mathrm{~mm}$ and $220 \mathrm{~mm}$ were slightly fuzzy. The trend on the results of resistance, adhesion tape test and micro hardness test show the ink track cured at $165 \mathrm{~mm}$ distance had a better resistance, adhesion and hardness. The temperature readings on the sample during curing are also at its highest in which it is mainly dependant on the sharpness of the image. Even though the $110 \mathrm{~mm}$ distance between the lens and platform is nearer to the samples, the results would not be any better. Such different distance is not important when the light image obtained is low in intensity.

Impact of a curing time. The curing time has been set up from 5 to $25 \mathrm{~min}$ with an increment of $5 \mathrm{~min}$. The results turned out to be as expected in which the longer curing time resulting in better performance of the ink track. The samples cured at three different distances showed different value but the entire trend showed positive effect as the curing time increased. The resistance of the ink track decreased as the curing time increased and it gives better conductivity. The level of adhesion obtained increased as the curing time increased. The adhesion level proved that the level of cured ink track grew as it is cured at a longer period of time. Micro hardness test results on samples cured at longer period of time were greater than the shorter time among their curing distance categories. These results showed that the longer curing time applied, the more energy is provided to strengthen the cross linking of the silver particles which harden the ink track. 


\section{Conclusion}

Silver conductive ink is the most preferable among the other types especially in RFID tags application. The market demands on the silver based ink have caused in various type of production improvement. There were two important elements that are able to be improved which is the setup cost and production time. Using DLP projector as light source for curing process, the cost is lowered compared to the laser technology. As in curing time, DLP projector shows shorter curing time to cure the conductive ink tracks rather than conventional oven which took up to 1 hour of curing time. In addition, DLP projector is capable of curing silver conductive ink and the correlation between the conductivity of the cured ink track and the parameters used in the experiment have been determined. The tape test and micro hardness test showed the curing level of the silver conductive ink. The distance at which the sharpest light image is obtained result in the highest curing level. The fact that the higher curing level had better conductivity of the ink track is proven by the resistance reading. The temperature readings taken during curing process proved that the sharpest light image provided higher light intensity which is sufficient enough to cure the silver conductive ink.

\section{Acknowledgement}

The authors greatfully acknowledge the support to the University of Tun Hussein Onn Malaysia under a short term grant awarded and giving the opportunity to attend and present at this conference.

\section{References}

[1] P. B. Kyun, K. Dongjo, J. Sunho, M. Jooho, K. J. Sub, Direct writing of copper conductive patterns by ink-jet printing. Thin Solid Films, 515 (2007) 7706-7711.

[2] L. K. Fiddes, N. Yan, RFID tags for wireless electrochemical detection of volatile chemicals, Sensors and Actuators B: Chemical, 186 (2013) 817-823.

[3] D. A. Roberson, A Novel Method for The Curing of Metal Particle Loaded Conductive Inks and Pastes, The University of Texas: Degree of Doctor of Philosophy (2012)

[4] S. Shang, D. Wellburn, E. Fearon, S. Yan, S. Edwardson, G. Dearden, K. Watkins, Laser assisted direct write process with novel beam profiles, Optics and Lasers in Engineering, 51(5) (2013) 527-532.

[5] L. Fu, S. Shang, E. Fearon, W. Perrie, S. Edwardson, G. Dearden, K. Watkins. A Thermal Investigation on Conductive Silver Ink Tracks Cured on Flexible Substrates by Repeating Irradiations of Nd:Yag Laser at The Wavelength of $532 \mathrm{Nm}$, University of Liverpool (2012).

[6] K. Kamarudin, I. Raman, M. A. S. Mohamed, M. Ibrahim, M. S. Wahab, Parameter Optimization For Photopolymerization Of Mask Projection Micro Stereolithography, Universiti Tun Hussein Onn Malaysia: Master Thesis (2013).

[7] E. Fearon, T. Sato, D. Wellburn, K. G. Watkins, G. Dearden, Thermal Effects of Substrate Materials used in The Laser Curing of Particulate Silver Inks, Laser Assisted Net Shape Engineering, 5 (2007) 379-390.

[8] S. B. Fuller, E. J. Wilhelm, J. M. Jacobson (2002), Ink-Jet Printed Nanoparticle Microelectromechanical Systems, Journal of Microelectromechanical Systems, 11(1) (2002) 54-60.

[9] X. Nie, H. Wang, J. Zou, Inkjet printing of silver citrate conductive ink on PET substrate, Applied Surface Science, 261 (2012) 554-560. 